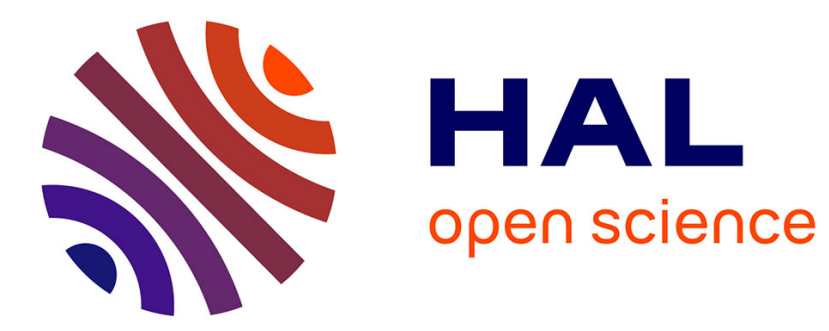

\title{
Automatic damage type classification and severity quantification using signal based and nonlinear model based damage sensitive features
}

Meriem Ghrib, Marc Rébillat, Guillaume Vermot Des Roches, Nazih Mechbal

\section{- To cite this version:}

Meriem Ghrib, Marc Rébillat, Guillaume Vermot Des Roches, Nazih Mechbal. Automatic damage type classification and severity quantification using signal based and nonlinear model based damage sensitive features. Journal of Process Control, 2019, 83, pp.136-146. 10.1016/j.jprocont.2018.08.002 . hal-03065251

\section{HAL Id: hal-03065251 \\ https://hal.science/hal-03065251}

Submitted on 14 Dec 2020

HAL is a multi-disciplinary open access archive for the deposit and dissemination of scientific research documents, whether they are published or not. The documents may come from teaching and research institutions in France or abroad, or from public or private research centers.
L'archive ouverte pluridisciplinaire HAL, est destinée au dépôt et à la diffusion de documents scientifiques de niveau recherche, publiés ou non, émanant des établissements d'enseignement et de recherche français ou étrangers, des laboratoires publics ou privés. 


\title{
Automatic damage type classification and severity quantification using signal based and nonlinear model based damage sensitive features
}

\author{
Meriem Ghrib ${ }^{\mathrm{a}}$, Marc Rébillat ${ }^{\mathrm{a}, *}$, Guillaume Vermot des Roches ${ }^{\mathrm{b}}$, Nazih Mechbal ${ }^{\mathrm{a}}$ \\ a PIMM laboratory, Arts et Métiers ParisTech, 75013 Paris, France \\ b SDTools, France
}

\begin{abstract}
Structural health monitoring (SHM) is an emerging technology designed to automate the inspection process undertaken to assess the health condition of structures. The SHM process is classically decomposed into four sequential steps: damage detection, localization, classification, and quantification. This paper addresses damage type classification and severity quantification issues as classification problems whereby each class corresponds to a given damage type or a certain damage extent. A Support Vector Machine (SVM) is used to perform multi-class classification task. Classically, Signal Based Features (SBF) are used to train SVMs when approaching SHM from a machine learning perspective. In this work, starting from the assumption that damage causes a structure to exhibit nonlinear response, it is investigated whether the use of Nonlinear Model Based Features (NMBF) increases classification performance. NMBF are computed based on parallel Hammerstein models which are identified with an Exponential Sine Sweep (ESS) signal. A study of the sensitivity of classification performance to the noise contained in output signals is also conducted. Dimension reduction of features vector using Principal Component Analysis (PCA) is carried out in order to find out if it allows robustifying the classification/quantification process suggested in this work. Simulated data on a cantilever beam with various damage types and severities as well as experimental data coming from a composite aeronautic plate with various damage severities generated with a unique and original laser process are considered for demonstration. For both application cases, results show that by introducing NMBF, classification performance is improved. Furthermore, PCA allows for high recognition rates while reducing features vector dimension.
\end{abstract}

\section{Introduction}

Structural Health Monitoring (SHM) [1-3] combines advanced sensor technology with intelligent algorithms to interrogate the health condition of monitored structures. SHM is of interest in many applications, including civil, mechanical, and aerospace engineering. Generally, the SHM process entails establishing: (1) the existence of damage, (2) the damage location, (3) the type of damage, and (4) the damage severity [4]. The focus is more particularly put here on the monitoring process of composite structures. A major difference between fault diagnosis and SHM lies in the fact that in fault diagnosis the monitored systems are by nature "discrete" in the sense that faults can appear on a limited number of

\footnotetext{
* Corresponding author.

E-mail address: marc.rebillat@ensam.eu (M. Rébillat).
}

components that compose the whole system whereas monitored structures are "continuous" in the sense that damage can appear anywhere within the structure. This renders the task of SHM much more multidisciplinary and challenging than fault diagnosis. Furthermore, accessing to experimental data that covers all damaged cases may not always be possible. Indeed, structural damages are very difficult to reproduce in a realistic manner on actual samples. Moreover, within the case of composite structures, the diversity of damage types and the lack of knowledge on their evolution make the use of only experimental data very difficult. Currently, several works deal with this challenging problem of generating controlled real damages with dedicated NDE systems and postmortem test to validate them [5]. As a consequence, a considerable amount of literature exists on damage detection and localization techniques [6-9]. However, very little research has been undertaken to respond to damage type classification and severity quantification issues. 
In [10] damage classification is performed using time-frequency representations and the Adaboost machine learning algorithm. In [11], damage type classification is transformed into a group classification process, under the influence of uncertainty. More recently, Vitola et al. [12] proposed a data-driven methodology for the detection and classification of damages by using multivariate data driven approaches and machine learning algorithms. In [13], the damage quantification problem is approached from a full wavefield perspective and through local wavenumber estimation. In a unsupervised learning framework, novel damage indices for damage quantification have been proposed recently in [14].

These approaches all have a common feature: they rely only on linear non model-based features as inputs to more or less evoluted machine learning algorithms. But in many cases, damages that appear on complex structures (such as cracks, impacts, or delaminations) can generate nonlinear dynamical responses that may be used for damage monitoring [15-18]. Furthermore, complex structures often exhibit a nonlinear behavior even in their healthy states. A robust and reliable SHM system must then be able to deal with nonlinear damages, and to distinguish between their effects and inherent nonlinearities in healthy structures. There have been some works in that direction, but never for damage classification or quantification purposes. In a linear framework, some authors $[19,20]$ have shown that a nonlinear damage will impact the transmissibility functions (i.e. the frequency domain ratio between two different outputs of the system) and they used such information to detect and locate the damage. Extending the notion of transmissibility functions to nonlinear systems that can be described by Volterra series, Lang et al. [21,22] were able to quantify the decrease of linearity generated by a nonlinear damage and thus to effectively detect and locate it. However, as such approaches are focusing on the loss of linearity, they do not seem to be able to deal with systems that are nonlinear in their healthy states, a fact that is quite common in real life. To overcome this drawback, several authors attempted to fit a nonlinear model to the nonlinear structure under study and to compare the actual and predicted outputs, or directly the model coefficients, under different damage conditions [23-26]. By doing so, they were able to detect numerically and experimentally a nonlinear damage even in an initially nonlinear structure. In [27], a relatively general nonlinear model (parallel Hammerstein model, belonging to the class of Volterra models) has been used to extract successfully nonlinear damage and structure related information for damage detection purpose.

In this article, we aim at exploiting a richer nonlinear representation of our test structure and at investigating whether the use of nonlinear model based features allows for an enhanced damage classification/quantification approach. More specifically, damage type classification and severity quantification issues are transformed into classification problems whereby each class corresponds to a given damage type or severity. A support vector machine is used to perform multiclass classification. Two types of features are used as inputs to the SVM algorithm: Signal Based Features (SBF) and Nonlinear Model Based Features (NMBF). SBF are rooted in a direct use of response signals and do not consider any underlying model of the structure under study. To compute NMBF, parallel Hammerstein models are considered to model the damaged structure. The model is identified using an Exponential Sine Sweep (ESS) [28-31] excitation signal and NMBF are afterwards computed based on the identified Hammerstein kernels. PCA has generally been used in SHM field as a technique to establish damage sensitive features [8,32]. In this work PCA is used to reduce the dimension of features vector, the aim being to find out if dimension reduction allows robustifying the suggested classification/quantification approach. Furthermore a study of the sensitivity of classification performance to the noise contained in output signals is performed. Simulation results on a realistic cantilever beam

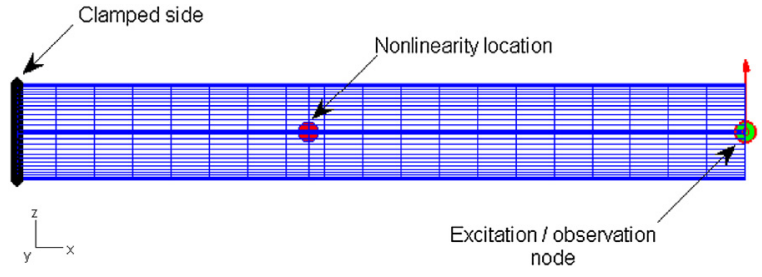

Fig. 1. Illustration of the simulated model. (For interpretation of the references to color in text near the figure citation, the reader is referred to the web version of this article.)

with various damage types and severities as well as experimental data coming from a composite plate representative of aeronautic applications with various damage severities generated with a unique and original laser process are considered as demonstration examples.

The remaining of the paper is organized as follows. Firstly, the test structure considered for demonstration is presented. Then, the main key ingredients of the classification/quantification approach suggested in this work are introduced. The application of the proposed approach to damage type classification is afterwards detailed. Simulation results of various damage types used to derive damage sensitive features are described and classification and quantification performances are presented for this application case. The application of the proposed approach to damage severity quantification is subsequently elaborated using experimental data. Conclusions and perspectives are finally drawn.

\section{Test structures}

\subsection{Beam simulated model}

The first test structure on which we conducted our study is a simulated beam model developed in a previous work for the investigation of a vibration-based structural health monitoring procedure on high voltage electrical switch insulators [33]. SDTools Matlab toolbox (SDT for Matlab) [34] is used to simulate the dynamic response of our model. The base model is a cantilever beam of length $4 \mathrm{~m}$ and circular section of radius $0.17 \mathrm{~m}$. The beam is in steel with properties $E=210 \mathrm{GPa}, v=0.285$ and $\rho=7800 \mathrm{~kg} / \mathrm{m}^{3}$. At a given nodal position a torsion spring is placed between the two rotational Degree Of Freedom (DOF) instead of the continuum coupling. The torsion spring stiffness (parameter $k_{v}$ ) is calibrated to be close to its saturation level, the threshold is defined as $95 \%$ of the maximum frequency of the first mode. Several non-linearities for the torsion spring stiffness are implemented to simulate various damage types. Four types of torsion spring stiffness nonlinearities are considered as shown through force-relative displacement curves (Fig. 2): bilinear stiffness, saturation, dead zone and Coulomb friction. The non-linearities are defined as relative to a healthy state for which the torsion spring behaves linearly. One defines a generic damage severity parameter $\alpha$ that should vary between 0 (healty) and 1 (totally damaged). A first simulation in the healthy state is also performed to define $R_{\max }$, the maximum relative displacement for the undamaged structure. The four nonlinearities have been chosen as idealized representations of four types of damage:

- The bilinear stiffness (Fig. 2(a)) physically corresponds to a crack that is opening and closing and thus applies a lower stiffness in traction. Compression stiffness is set to $k_{v}$. Traction stiffness is set to $(1-\alpha) k_{v}$.

- The saturation (Fig. 2(b)) can be seen as a dry friction phenomenon associated with a spring and may correspond to relative movements between parts of a system caused by an overdrive in 


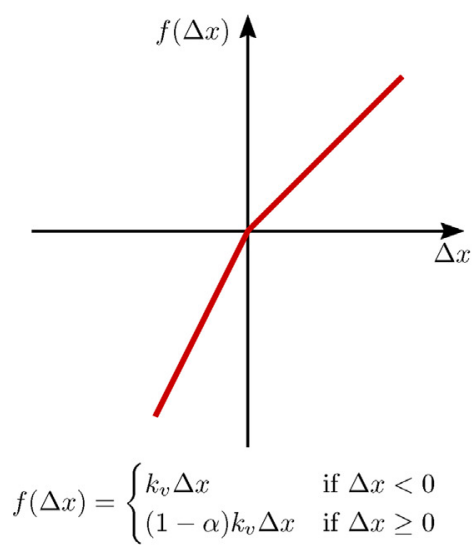

(a)

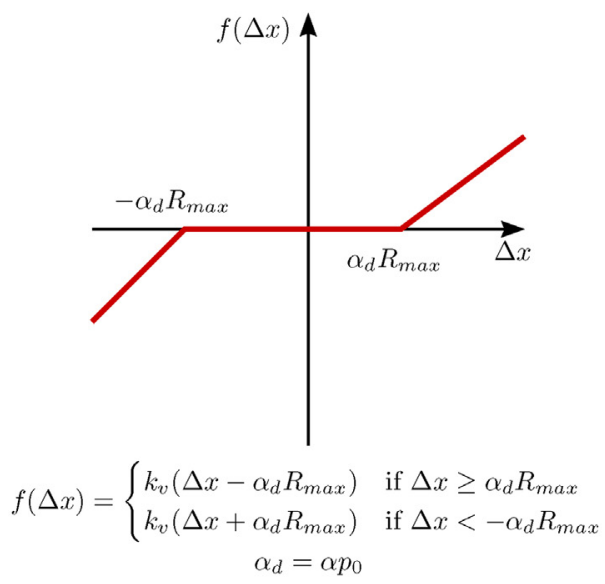

(c)

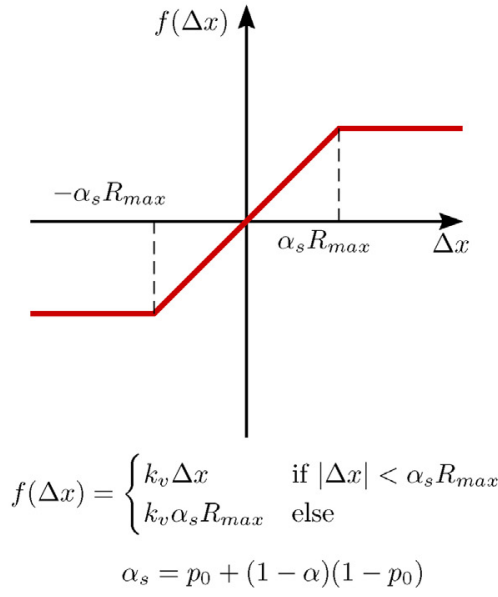

(b)

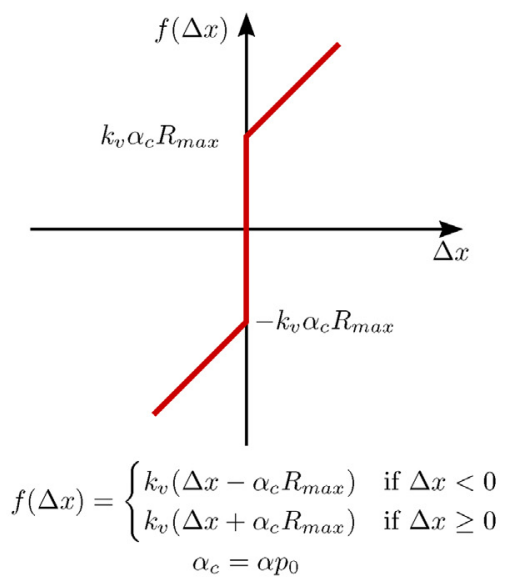

(d)

Fig. 2. Stiffness non-linearities illustrated: (a) bilinear, (b) saturation, (c) dead zone, (d) Coulomb friction.

force. The base stiffness is set to $k_{v}$, the saturation occurs for a maximum relative displacement set to $\left(p_{0}+(1-\alpha)\left(1-p_{0}\right)\right) R_{\max }$. Thus, for $\alpha$ equal to 0 , there is no saturation and for $\alpha$ equal to 1 , a saturation occurs at a relative displacement of $p_{0} R_{\max }$. Hence, $p_{0}$ can be seen as a percentage of saturation.

- The dead zone models systems where damage causes some mechanical slack without any contact between two parts. The dead zone (Fig. 2(c)) applies to the torsion spring a linear stiffness $k_{v}$ only outside a relative displacement threshold, defined as $\alpha p_{0} R_{\max }$. Thus, for $\alpha$ equal to 0 , there is no dead zone and for $\alpha$ equal to 1 , a dead zone extends over a relative displacement range of $-p_{0} R_{\max }$ to $p_{0} R_{\max }$. Hence, $p_{0}$ can be seen as a percentage defining the extent of the dead zone.

- The Coulomb friction models systems where damage manifests itself by the appearance of Coulomb friction due for example to the lack of lubrication between parts. It consists of a brutal force sign switch (Fig. 2(d)) and applies the linear torsion spring stiffness, with a force offset between traction and compression. The offset occurs for relative displacements under a tolerance of $10^{-6}$ and it is generated by considering an offset of $\alpha p_{0} R_{\max }$ in the relative displacement observation. Thus, for $\alpha$ equal to 0 , there is no force offset and for $\alpha$ equal to 1 , the force goes from $-k_{v} p_{0} R_{\max }$ to $p_{0} R_{\max }$. Hence, $p_{0}$ can be seen as a percentage defining the Coulomb friction.

\subsection{Aeronautic composite plates}

The second test set of test structures considered for demonstration consists of CFRP composite plates equipped with piezoelectric elements and containing various delamination severities. Damage is introduced into samples (see Fig. 3) in a calibrated way using Laser Shock Wave Technique (LSWT) and more particularly symmetrical laser shock configuration. LSWT is chosen as an alternative to conventional damage generation techniques such as conventional impacts and Teflon inserts since it allows for a better calibration of damage in type, depth and size [35,5,36]. Four CFRP test coupons are considered. The first one is kept in a healthy state. The second one was subjected to a symmetrical laser impact at 0 ns time delay and at $100 \%$ of the maximum energy of the two laser beams [36]. This resulted in approximately $7 \mathrm{~mm}$ diameter delamination which occurred at $1.1 \mathrm{~mm}$ depth. The third coupon was subjected to two symmetrical and contiguous laser impacts which resulted in $14 \mathrm{~mm}$ diameter delamination while the fourth coupon was impacted with three contiguous impacts which in turn resulted in approximately $21 \mathrm{~mm}$ diameter delamination. A photography and a C-Scan analysis of the four coupons is provided in Fig. 4 highlighting the fact that generated damaged exists but are not visible. 


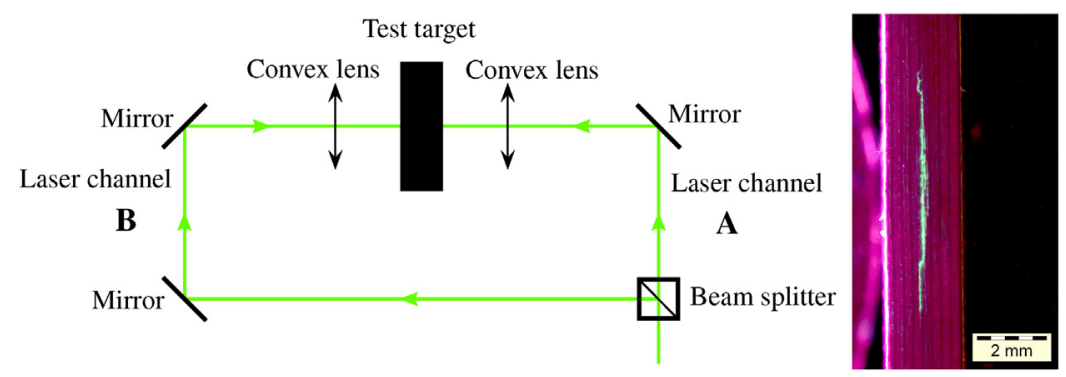

Fig. 3. Left: Experimental set-up of symmetrical laser shock configuration. Right: Cross-sectional observation showing a delamination generated using LSWT.
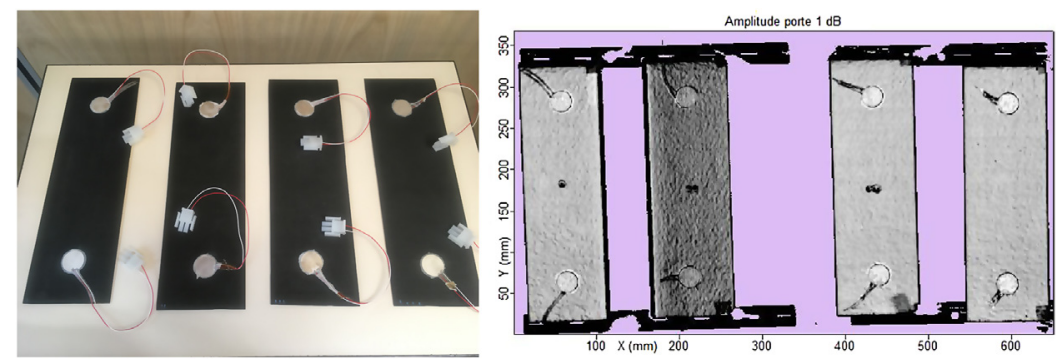

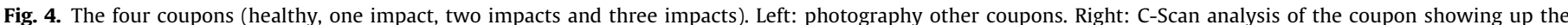
delamination generated using LSWT (from left to right: one impact, two impacts, three impacts and healthy).

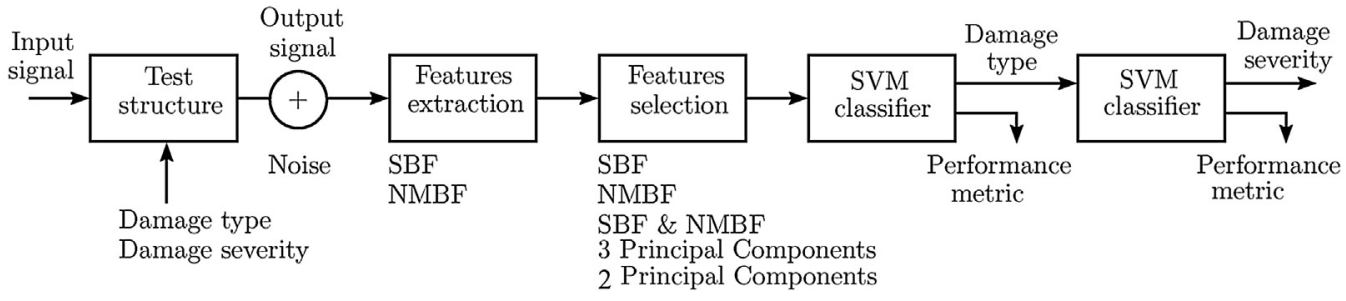

Fig. 5. Workflow suggested for damage classification/quantification.

\section{Proposed damage classification/quantification approach}

Fig. 5 illustrates the main key ingredients of the classification/quantification workflow proposed in this work. An input signal is firstly selected to excite a test structure containing a given damage type and severity. The structure response signal is then recorded and damage sensitive features are extracted. In this work, the first question which arises is whether NMBF allow for an enhanced damage classification/quantification strategy on the two application cases previously described. Two types of features are thus considered: SBF and NMBF.

\subsection{Excitation signal}

The input signals considered in this work are a punctual force in the $z$ direction for the cantilever beam (red arrow in Fig. 1) and an electrical excitation of a piezoelectric element for the composite plate whose amplitudes are defined by an exponential sine sweep curve. Various parameters are specified to define the input sweep signals for each considered application cases:

- the minimum frequency $f_{\min }$ (set to $0.7 \mathrm{~Hz}$ for the beam and to $1 \mathrm{kHz}$ for the composite plate)

- the maximum frequency $f_{\max }$ (set to $25 \mathrm{~Hz}$ for the beam and to $100 \mathrm{kHz}$ for the composite plate)

- the sampling frequency $f_{s}$ (set to $110 \mathrm{~Hz}$ for the beam and to $1 \mathrm{MHz}$ for the composite plate)
- the sweep duration $t_{\text {length }}$ (set to $180 \mathrm{~s}$ for the beam and to $0.45 \mathrm{~s}$ for the composite plate)

- the sweep amplitude amp (set to $1000 \mathrm{~N}$ for the beam and to $10 \mathrm{~V}$ for the composite plate)

\section{2. $S B F$}

Signal based features are rooted in a direct use of response signals and do not consider any underlying model of the test structure. Four signal-based features are considered in this study and are computed as follows. Let $s_{r e f}(t)$ and $s_{d}(t)$ be the structure output signal in reference and damaged state respectively, where $t$ refers to time, one defines the SBF as in Table 1.

\section{3. $N M B F$}

NMBF are considered based on previous work presented in $[37,8]$. Parallel Hammerstein models are selected to model the damaged structure (see Fig. 6). The reason behind this choice is that such model is sufficiently general and it allows to capture all or part of damage generated nonlinearities. Furthermore, Parallel Hammerstein models are easy to estimate and to interpret. The model is fully represented by its kernels $\left\{h_{n}(t)\right\}_{n \in\{1, \ldots, N\}}$ (N being the model order and can be automatically estimated [31]) and is identified by means of Exponential Sine Sweeps excitation signal 
Table 1

Signal based features.

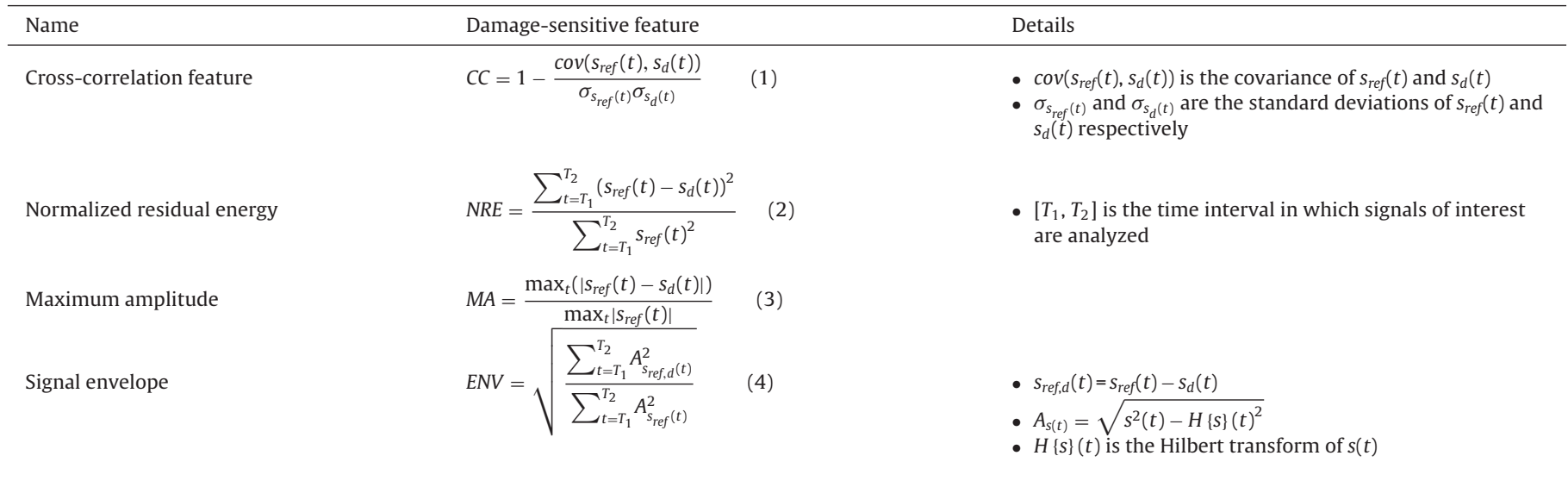

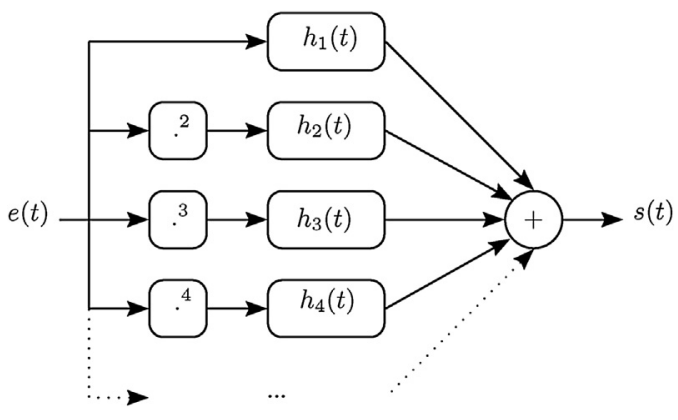

Fig. 6. Parallel Hammerstein models.

$e(t)$. Once the model is identified, the system output $s(t)$ can be rewritten as follows:

$s(t)=\sum_{n=1}^{N}\left(h_{n} * e^{n}\right)(t)$

$=\left(h_{1} * e\right)(t)+\sum_{n=2}^{N}\left(h_{n} * e^{n}\right)(t)$

$=s^{L}(t)+s^{N L}(t)$

$=s^{L}(t)+\sum_{n \text { odd }}\left(h_{n} * e^{n}\right)(t)+\sum_{n \text { even }}\left(h_{n} * e^{n}\right)(t)$

$=s^{L}(t)+s_{o}^{N L}(t)+s_{e}^{N L}(t)$

The output signal is decomposed into a linear part and a nonlinear part (6). The nonlinear part is in turn decomposed into odd harmonics contribution and even harmonics contribution (8). Three Non Linear Model Based Features (NMBF) are defined and computed as detailed in Table 2.

\subsection{SVM and PCA}

SVMs Support vector machine learning technique is used, in this work, for classification. SVMs [38] are originally introduced by Vapnik and co-workers $[39,40]$ and successfully extended by a number of other researchers. SVM learning algorithm separates a given set of binary labeled training data with a hyper-plane that is maximally distant from them (known as the maximal margin hyper-plane). For cases in which no linear separation is possible, they can work in combination with the technique of "kernels", that automatically realizes a non-linear mapping to a feature space. The hyper-plane found by the SVM in the feature space corresponds to a non-linear decision boundary in the input space. To extend SVMs to multi-class scenario, a typical conventional way is to decompose a multi-class problem into a series of two-class problems. One can distinguish between two implementations:

- One Against All "OAA" approach

- One Against One "OAO” approach

The "OAO" and the "OAA" are two popular strategies for multiclass SVM. "OAO" builds one SVM for each pair of classes while "OAA" consists of building one SVM per class, trained to distinguish the samples in a single class from the samples in all remaining classes. In this work, a Gaussian kernel SVM is considered. SVM and Kernel Methods (SVM-KM) Matlab toolbox [41] is used to perform multiclass classification.

PCA Principal component analysis (PCA) [42] is a popular tool for linear dimensionality reduction and feature extraction. Intuitively, PCA can supply the user with a lower-dimensional picture of data when viewed from its most informative viewpoint. Several extensions of the standard PCA have been proposed such as the Kernel PCA which is the nonlinear form of PCA and which better exploits the complicated spatial structure of high-dimensional features. In this work we opted for the standard PCA since our features vector is not very high-dimensional.

\subsection{Input features scenarios}

As mentioned previously, the two main issues addressed in this paper consist in finding out if the use of nonlinear model-based features increases classification performance and whether reducing features vector dimension using PCA allows robustifying the suggested classification/quantification approach. Several input features scenarios are, thus, considered according to which features to select to feed and train the SVM algorithm:

- Scenario 1: Only SBF are used to train the SVM algorithm

- Scenario 2: Only NMBF are used to train the SVM algorithm

- Scenario 3: Both SBF and NMBF are used to train the SVM algorithm

- Scenario 4: PCA is performed on both SBF and NMBF and only 2 principal components are used to train the SVM algorithm

- Scenario 5: PCA is performed on both SBF and NMBF and only 3 principal components are used to train the SVM algorithm 
Table 2

Nonlinear model based features.

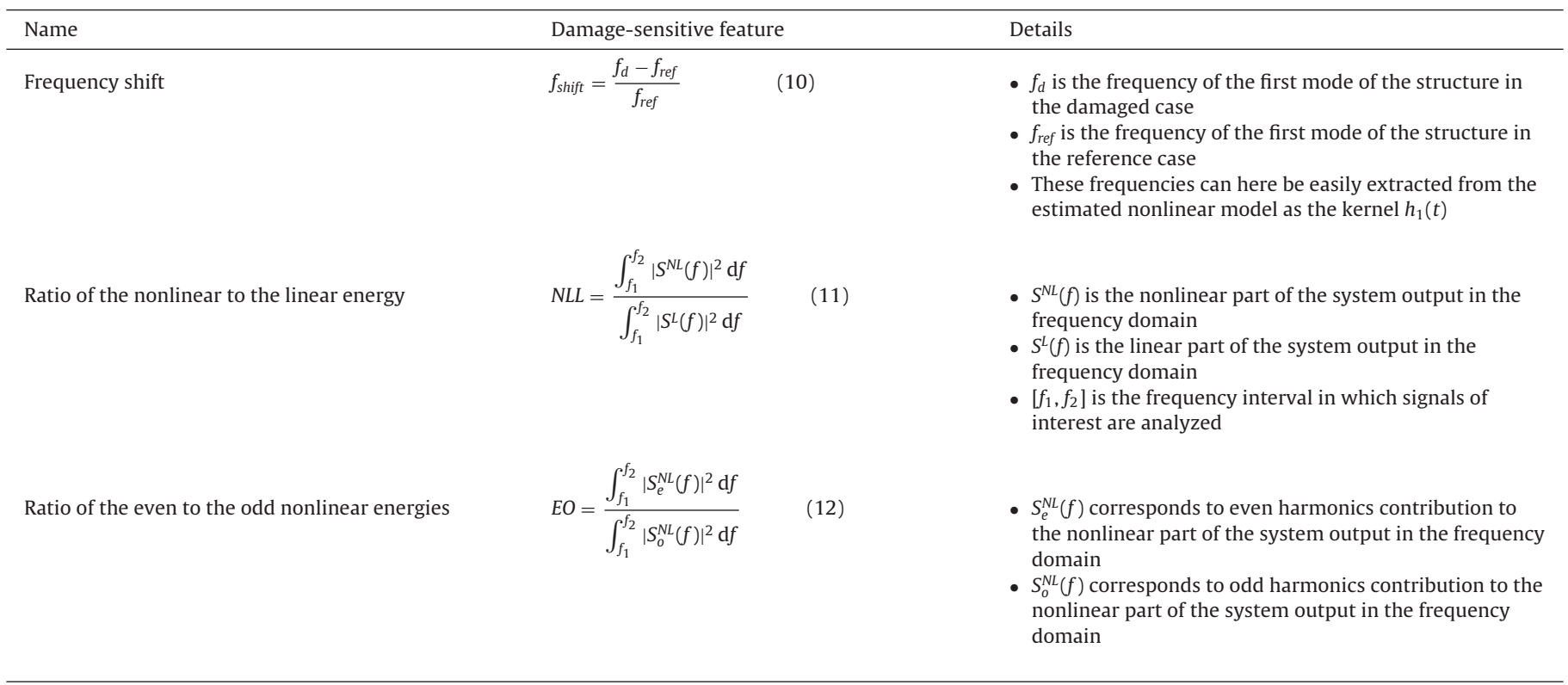

\section{Results for the simulated beam model}

\subsection{Damage type classification}

\subsubsection{Simulation data}

The actual application case behind the proposed methodology is for an industrial end user to be able to assess when its structure is "damaged enough" so that maintenance is mandatory in order to guarantee safety. Having an exact knowledge of damage severity is not what is sought here and the objective is more to be able to classify the damaged state among the categories "low", "mid" or "severe" for example, than to provide an exact damage severity value. This is relevant in the context of predictive maintenance and not in the context of estimation of the remaining useful life of the component under study. The discrete damage severity categories have been chosen on a relatively fine grid in order to get a correct coverage of all the damaged states of the structure without having too many damage categories. Thus, for each damage type, simulations are done with damage severities varying from 0 to 0.9 with steps of 0.15 . Each damage severity is considered at damage location NLpos equal to 0.5 . Setting NLpos to a given value $v, v \in[0,1]$, stands for a damage position at $v \times 100 \%$ of the beam length from the clamped side. The position of the excitation force is set to 1 , standing for $100 \%$ of the beam length from the clamped side. The position of the observation node is set at the excitation location. After running simulations, white Gaussian noise with SNR (Eq. (13)) varying from $40 \mathrm{~dB}$ to $100 \mathrm{~dB}$ by steps of $5 \mathrm{~dB}$ is added to the output signals.

$S N R_{d B}=10 \log _{10}\left(\frac{P_{\text {signal }}}{P_{\text {noise }}}\right.$

where $P_{\text {signal }}$ and $P_{\text {noise }}$ are output signal and noise power respectively.

In order to enrich our features database, 20 repetitions were considered for each SNR value. With such parameters, a total of 7280 noisy output signals are obtained; 1820 noisy outputs for each class of damage type. The output signals obtained at this step are used to compute damage sensitive features. Thus, one obtains 1820 instances for each class of damage type. Various classifiers are afterwards trained according to SVM input features scenarios (see Section 3.5). For training, only damage sensitive features computed
Table 3

Coefficients for principal components in original damage-sensitive features space.

\begin{tabular}{llll}
\hline & PC1 & PC2 & PC3 \\
\hline CC & 0.4497 & -0.0885 & 0.0267 \\
$N R E$ & 0.4247 & -0.1023 & 0.1891 \\
$M A$ & 0.4248 & -0.1520 & -0.1344 \\
$E N V$ & 0.4515 & 0.0182 & 0.1022 \\
$f_{\text {shift }}$ & 0.4422 & -0.0564 & 0.0249 \\
$N L L$ & 0.0912 & 0.8262 & 0.5170 \\
EO & -0.1708 & -0.5220 & 0.8167 \\
\hline
\end{tabular}

using output signals with a maximum $S N R\left(S N R_{\max }=100 \mathrm{~dB}\right)$ are used. We assume that for training we consider the most favourable case where noise is very low. For real applications this may correspond to learning via models or in well-controlled environments. For test we consider less favourable situations where output noise is not negelected. Thus features computed using output signals with $S N R$ lower than $S N R_{\max }$ are used to test the classifiers.

\subsubsection{Classification results}

Fig. 7(a) and (b) illustrate, respectively, the recognition rate of various "OAO" and "OAA" multiclass classifiers on test data (in\%) versus SNR (in $d B$ ) while addressing damage type classification issue. It can be seen that classifiers trained on NMBF or both SBF and NMBF have the same performances for all values of SNR. Furthermore, for high signal to noise ratios (namely SNR greater than $70 \mathrm{~dB}$ ), those classifiers have the best performance in terms of classification rate on test data. The performance of classifiers trained on the first three principal components, which retain $95.4 \%$ of data variance, is slightly less effective than the performance of NMBF trained classifiers. However, such classifiers are more robust to noise. The same applies to the classifiers trained on the first two principal components which retain $83.3 \%$ of data variance. Such classifiers perform less well than three principle components trained classifiers. However, they are more robust to noise compared to NMBF trained classifiers. Finally, SBF trained classifiers rank last among all classifiers but do not show any sensitivity to output noise. Table 3 provides coefficients for the first three principal components in original damage-sensitive features space. For the first principal component, a nearly-equal contribution of $C C$, $N R E, M A, E N V$ and $f_{\text {shift }}$ is noted. For the second and third Princi- 


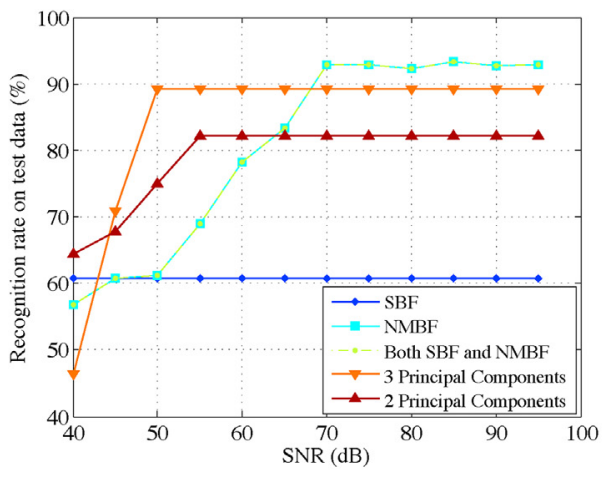

(a)

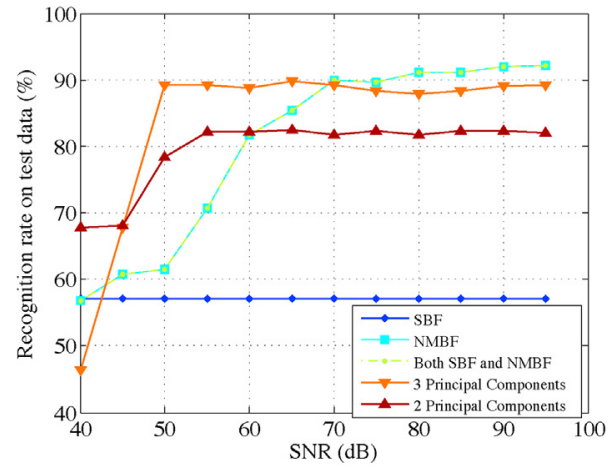

(b)

Fig. 7. Comparison of classification performance for various features scenarios - Damage type classification: (a) "OAO” approach, (b) "OAA" approach.

pal components, one can note a dominant contribution of $N L L$ and EO compared to all other damage sensitive features. Thus, one can draw from this that $N L L$ and $E O$ bring forward information with regard to damage type and, consequently, are very discriminant features.

\subsection{Damage severity quantification}

\subsubsection{Simulation data}

For damage quantification issue, one sets a specific damage type among "saturation", "dead zone" or "Coulomb friction" nonlinearities. The case of "bilinear" nonlinearity has already been dealt with in a previous IFAC paper [36]. Once the damage type fixed and with the simulation parameters detailed above, a total of 1820 noisy output signals are obtained: 260 noisy outputs for each class of damage severity. The output signals are afterwards used to compute damage sensitive features. Thus, one obtains 260 instances for each class of damage severity. Similarly, various classifiers are trained according to SVM input features scenarios (See Section 3.5). Fig. 8 plots output signals amplitude as a function of damage severity in the case of "dead zone" nonlinearity. It can be seen that an increase in damage severity results in greater distortions of output signals. This also applies for the other types of nonlinearities.

\subsubsection{Classification results}

Fig. 9(a) and (b) illustrate, respectively, the recognition rate of various "OAO" and "OAA" multiclass classifiers on test data (in\%) versus SNR (in $d B$ ) while addressing damage severity quantification issue for dead zone nonlinearity. Classifiers trained on the first two

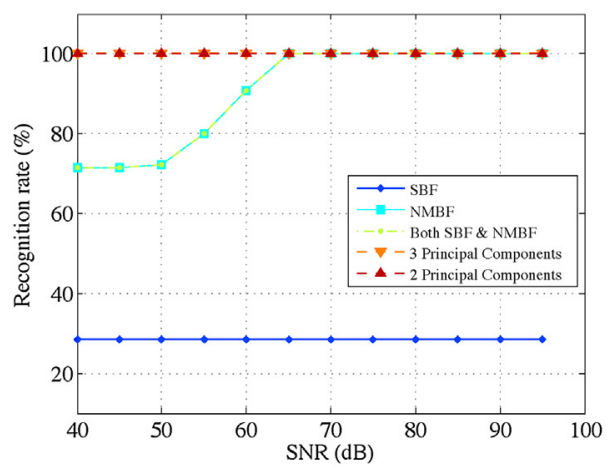

(a)

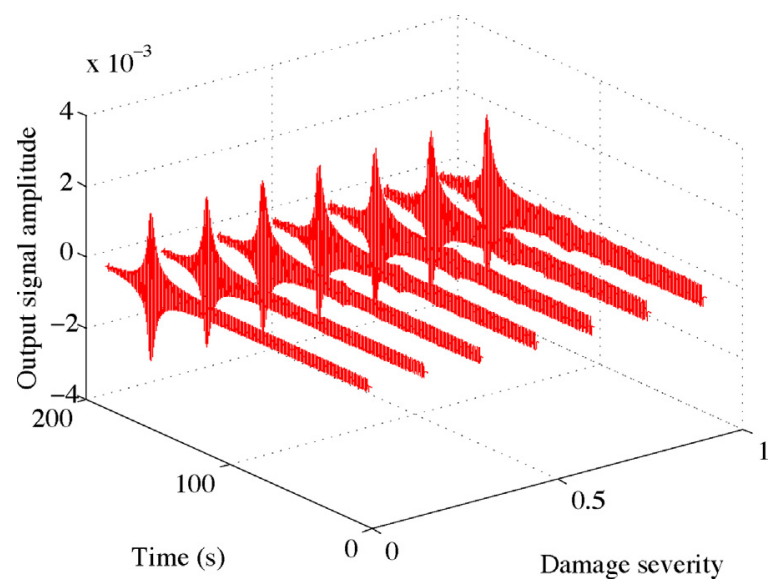

Fig. 8. Output signal amplitude for increasing damage severity - case of dead zone nonlinearity.

or three principal components are the best classifiers in terms of test data recognition rate. Furthermore, they show good robustness to output noise. NMBF trained classifiers have good performance for high values of SNR but they are very sensitive to output noise. Classifiers trained on SBF show poorer performance than all other classifiers but are robust to noise. All these remarks also apply to the cases of "Coulomb friction" and "saturation" nonlinearities (Figs. 10 and 11).

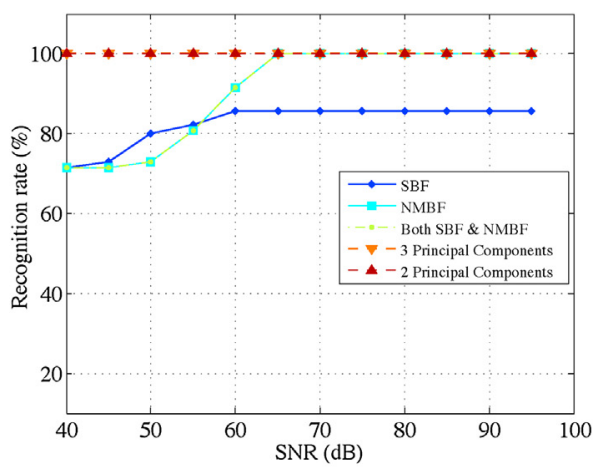

(b)

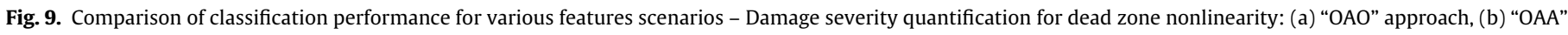
approach. 


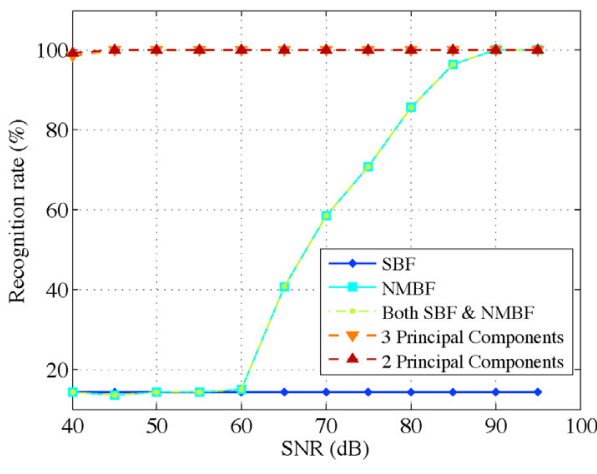

(a)

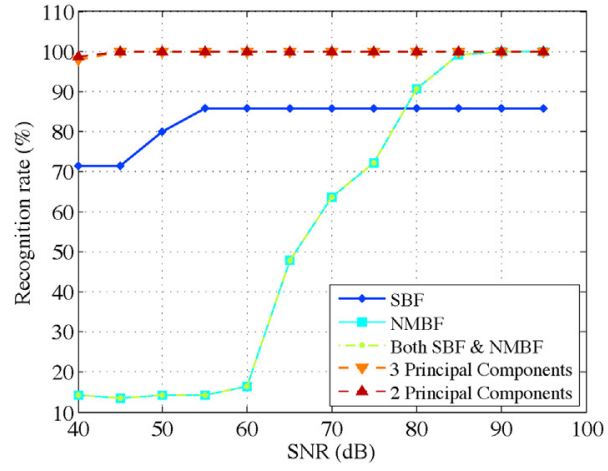

(b)

Fig. 10. Comparison of classification performance for various features scenarios - Damage severity quantification for Coulomb friction nonlinearity: (a) "OAO" approach, (b) "OAA" approach.

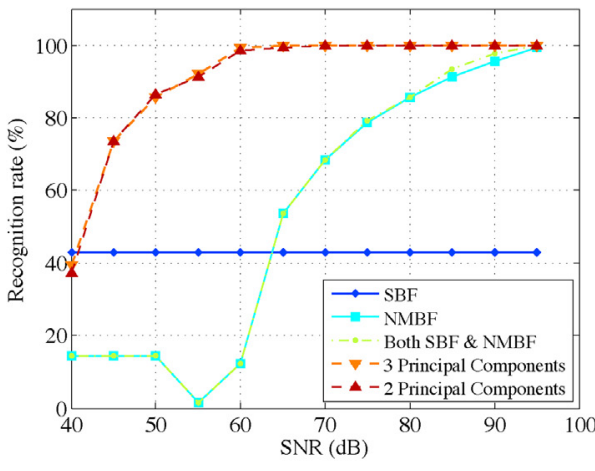

(a)

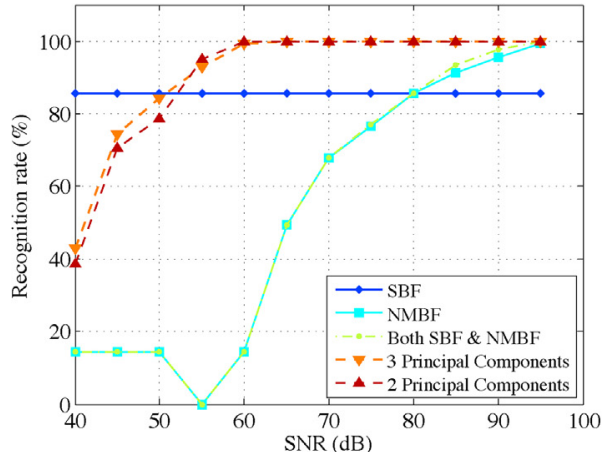

(b)

Fig. 11. Comparison of classification performance for various features scenarios - Damage severity quantification for saturation nonlinearity: (a) "OAO" approach, (b) "OAA" approach.
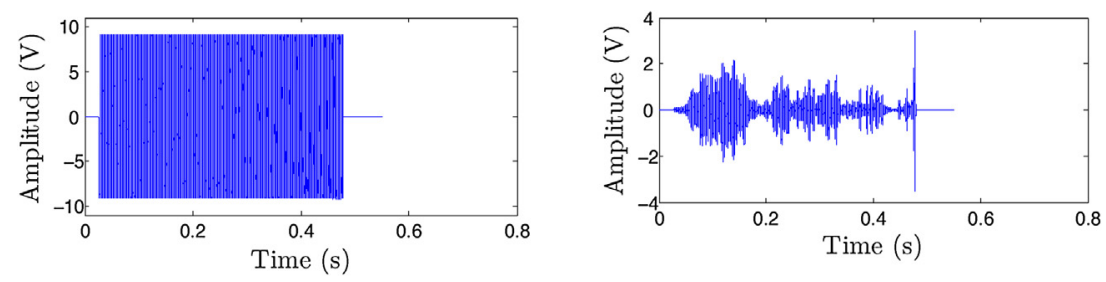

Fig. 12. Left: Excitation ESS signal, Right: Structure response signal.

\section{Results for the composite aeronautic plate}

\subsection{Experimental signals}

Twenty experimental repetitions are considered. At each repetition one goes around all PZT elements and only one PZT operates as an actuator while others operate as sensors. Acquisition frequency was set to $1 \mathrm{MHz}$. An instance of measurements corresponding to a given repetition is illustrated in Fig. 12.

\subsection{From output signals to input features}

Once signals are acquired, we proceeded to information condensation, that is, to damage-sensitive features computing. Each class of damage state ( 0 : healthy, 1 : one laser impact, 2 : two contiguous laser impacts and 3: three contiguous laser impacts) has 400 instances. Each instance is characterized by a total of seven features. Stratified sampling is considered; $70 \%$ of data is used for training the SVM model and $30 \%$ of data is used for testing the model. In the following figures, we represent training data in SBF space, NMBF space and in principal components spaces. The first three principal components retain $94 \%$ of data variance while the first two principal components retain $78 \%$ of data variance. Figs. 13 and 14 reveal a structure of four classes. Each corresponds to a state of damage severity. However, class separability is much better in NMBF space and in principal components spaces than in SBF space.

\subsection{Classification performance}

Ten SVM models are established according to which approach is adopted ('OAO' or 'OAA') and to which features are chosen as inputs (SBF, NMBF, SBF \& NMBF, 3 PCs or 2 PCs). Models performance on test data is then assessed. As illustrated in Fig. 15, independently of which approach is used, SVM models trained on NMBF or on principal components perform better than those trained on SBF. 

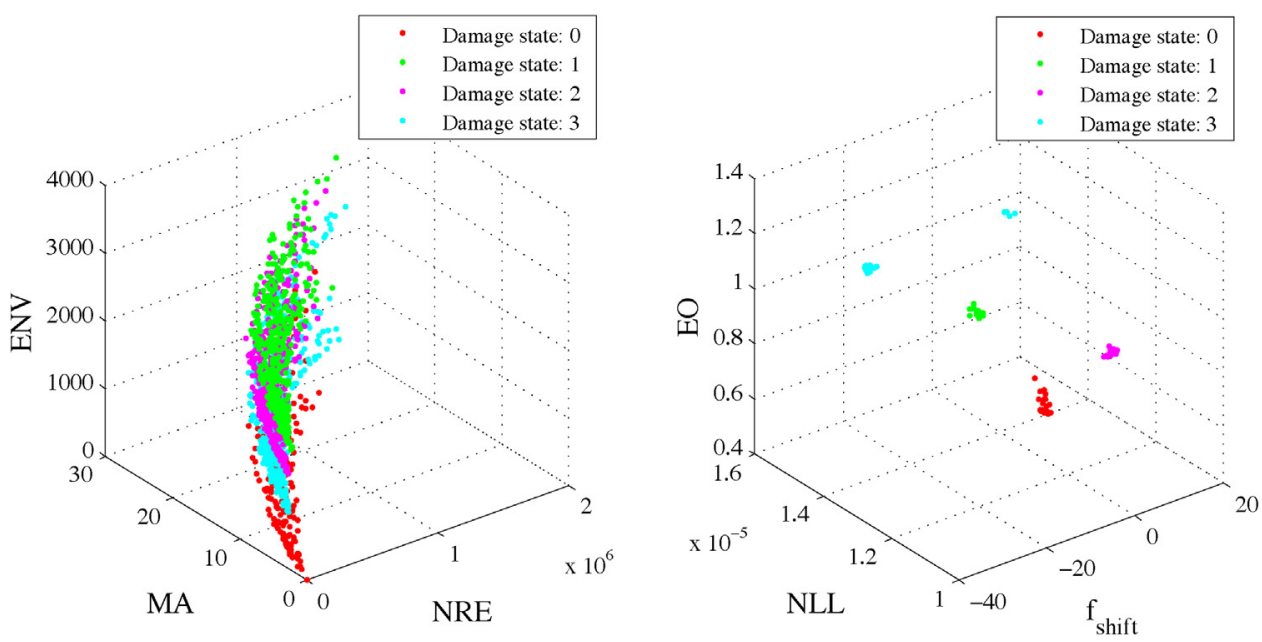

Fig. 13. Data representation - Left: in SBF space, Right in NMBF space.
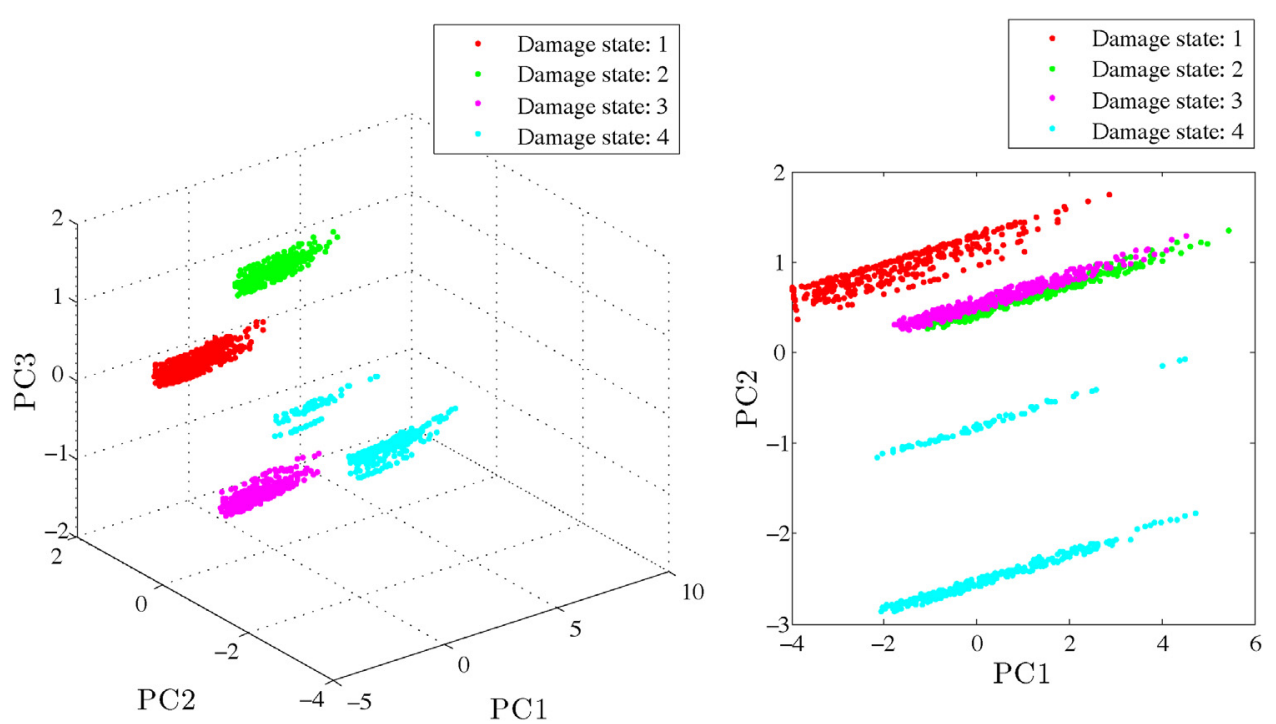

Fig. 14. Data representation in principal components spaces.

This is clearly due to class overlapping introduced by SBF and which induces the classifiers into error.

\section{Discussion}

\subsection{Limits of supervised damage classification and quantification algorithms}

One important point to mention here is that the damage classification and quantification methods proposed here both belong to the class of "supervised" learning algorithms. This means that, in opposition to "unsupervised" learning algorithms, data in the damaged cases are needed for the learning phase in both the damage classification and quantification stages.

The first point to discuss here is whether simulated data, which are much more easier to access than experimental data, may be sufficient to train the damage classification and quantification algorithm. Indeed, it is true that simulation models may not represent exactly real-life data and thus that training on real data may be mandatory to design robust damage classification and quantification algorithms. However, accessing to experimental data in a damaged case is not always possible, and merely impossible in most of the cases. In particular, designing a full experimental plan for various severities and all damage cases will be very costly and

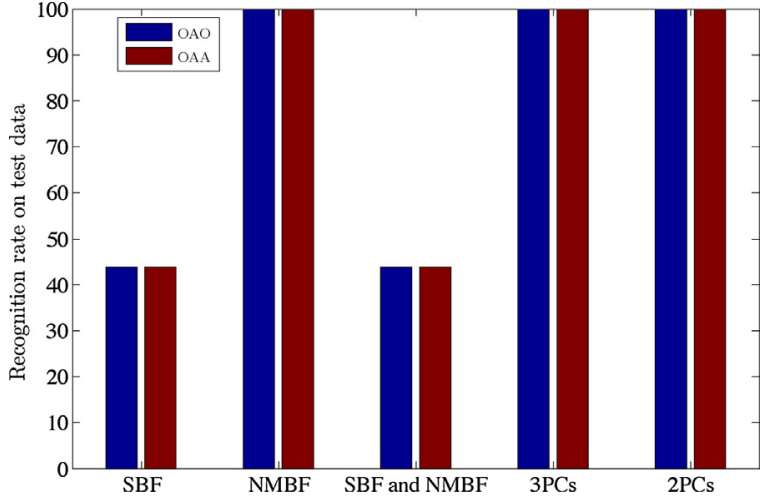

Fig. 15. Performance assessment of the considered SVM models on test data.

time consuming. However, in order to relax this assumption, it can be assumed that the effect of the damage type (related to the damage classification task) may be a qualitative change of the nonlinear dynamical behavior of the structure and thus may be correctly qualitatively described by the simulation model, and as a consequence may be sufficient for learning. Obviously, as no experimental data are accessible here, this point cannot be justified here 
and remains an idea to be validated. Furthermore, the quantification process is quantitative by nature and it is thus expected that even sophisticated simulation models may hardly scale exactly as the experimental specimens. As a consequence, experimental data for various damage level seems to be more mandatory as a basis for damage quantification algorithm learning. This is the process that has been followed here, but still damage quantification algorithm learning through simulated data remains a point that can be investigated.

\subsection{More optimal alternatives to principal component analysis}

In the proposed approach, Principal Component Analysis (PCA) has been used as a dimension reduction technique in order to investigate whether the information contained within all the damage indexes can be condensed to a lower dimensional space without loosing its classification abilities. However, as reported in [43], the aim of PCA is to find directions that explained the maximum of variance in the input data. One should here recall that in addition to input data, output data are also available as the classification problem is set up as a supervised classification problem here (see previous Section). There is thus a lot to learn with respect the dimension reduction directions that may be the more efficient for discrimination, as the goal is here indeed discrimination. In order to achieve this goal, some methods have been proposed such as Partial Least Squares (PLS) and Canonical Correlation Analysis (CCA) as well as their nonlinear kernel extensions [44-46]. Using one of these approaches in the present context is thus more appropriate than relying on standard PCA. However, when looking at the results obtained using PCA, which are extremely good, the gain in classification accuracy expected from PLS and CCA for the present example should be relatively minor. Nevertheless, using these methods for damage classification and quantification purposes remains a very relevant perspective. The multiclass SVM component, kernel selection and variable selection could also be further developed, as for example the multiple cores (MKL) and PLS discrimination or SVMs with integrated variable selection as in adaptive scaling.

\subsection{Balanced efficiencies between SBF and NLMBF with decreasing SNR}

The simulation results highlight the fact that when both SBF and NMBF are used for classification, the performance is identical to using NMBF alone with high SNR values. This suggests that the information coming from NMBF is dominant for these cases of SNR. However, the PCA augmented approach show significant improvement for low SNR situations, which suggest that SBF plays a dominant role for low SNR. It is hypothesized here that as NLBF rely on a model of the structure under study, then they allow to access to some "knowledge" about the structure under study, and thus that they are able to provide quite reliable information with respect to its current damage state. When dealing with SBF, the structure is not really modeled but the procedure is just an attempt to check out whether there is some strange behavior of one arbitrarily chosen attribute of the signal. As a consequence, it is to be believed that by nature SBF features carry less interesting information than NMBF features, which explains why NMBF features perform better as high SNR values. However, in order to extract reliable NMBF features, a reliable model is needed. Unfortunately, the estimated model quality degrades relatively quickly as SNR decreases [47]. Thus, when SNR is decreasing, the NMBF features become less and less reliable in comparison with SBF. This, in our opinion, explains the observed trends.

\section{Conclusions and perspectives}

In this paper, damage type classification and severity quantification issues are dealt with as classification problems whereby each class corresponds to a given damage type or a certain damage extent. SVM learning algorithm is used to perform multi-class classification task. Two main issues are addressed and consist in finding out if the use of nonlinear model-based features increases classification performance and whether reducing features vector dimension using PCA allows robustifying the suggested classification/quantification approach. A study of the sensitivity of classification performance to the noise contained in output signals is also conducted. From the outcome of our investigation it is possible to conclude that:

- For high values of SNR, NMBF bring forward more information on damage severity and type. Thus by introducing such features within the inputs of the SVM classifiers, classification performances are significantly improved. This applies to both "OAO" and "OAA" approaches.

- For high values of SNR and by performing PCA on both SBF and NMBF, classification performances are improved. Thus, PCA allows getting higher recognition rates on test data while reducing the dimension of features vector. Furthermore principal components trained classifiers show a higher robustness to noise compared to NMBF classifiers.

- For low values of SNR, it is necessary to take a step back with regard to the use of NMBF trained classifiers because it has been shown that their performance may be degraded.

- Classifiers trained on SBF show poorer performance than all other classifiers but are robust to noise.

As perspectives, the multiclass SVM component, kernel selection and variable selection could be further developed, as for example the multiple cores (MKL) and PLS discrimination or SVMs with integrated variable selection as in adaptive scaling.

\section{References}

[1] W. Staszewski, C. Boller, G.R. Tomlinson, Health Monitoring of Aerospace Structures: Smart Sensor Technologies and Signal Processing, first ed., Wiley, 2004.

[2] C.R. Farrar, K. Worden, An introduction to structural health monitoring, Philos. Trans. R. Soc.: B (2007), 365arker:303-15.

[3] C. Boller, F.K. Chang, Y. Fujino, Encyclopedia of Structural Health Monitoring, Wiley, 2009.

[4] A. Rytter, Vibrational Based Inspection of Civil Engineering Structures, (PhD thesis), University of Aalborg, 1993.

[5] M. Ghrib, L. Berthe, M. Rébillat, N. Mechbal, M. Guskov, R. Ecault, Laser shock a novel way to generate calibrated delamination in composites: concept and first results, in: 8th European Workshop On Structural Health Monitoring, Bilbao, Spain, 2016

[6] P.T. Coverley, W. Staszewski, Impact damage location in composite structures using optimized sensor triangulation procedure, Smart Mater. Struct. 12 (2003) 795-803.

[7] M. Vergé, N. Mechbal, R. Hajrya, Active damage detection and localization applied to composite structure using piezoceramic patches, in: Conference on Control and Fault Tolerant Systems, Nice, France, 2010.

[8] R. Hajrya, N. Mechbal, Principal component analysis and perturbation theory based robust damage detection of multifunctional aircraft structure, Struct. Health Monit. - Int. J. 12 (3) (2013) 263-277.

[9] C. Fendzi, N. Mechbal, M. Rébillat, M. Guskov, A general Bayesian framework for ellipse-based and hyperbola-based damage localisation in anisotropic composite plates, J. Intell. Mater. Syst. Struct. 27 (2016) 350-374.

[10] D. Kim, M. Philen, Damage classification using adaboost machine learning for structural health monitoring, in: Sensors and Smart Structures Technologies for Civil, Mechanical, and Aerospace Systems 2011, vol. 7981, International Society for Optics and Photonics, 2011.

[11] Z. Mao, M. Todd, Structural damage classification comparison using support vector machine and Bayesian model selection, 7th European Workshop on Structural Health Monitoring, vol. 197 (2014) 3-1980.

[12] J. Vitola, D. Tibaduiza, M. Anaya, F. Pozo, Structural damage detection and classification based on machine learning algorithms, 8th European Workshop On Structural Health Monitoring (2016, July). 
[13] O. Mesnil, C. Leckey, M. Ruzzene, Instantaneous and local wavenumber estimations for damage quantification in composites, Struct. Health Monit. 14 (3) (2015) 193-204

[14] A. Entezami, H. Shariatmadar, An unsupervised learning approach by novel damage indices in structural health monitoring for damage localization and quantification, Struct. Health Monit. 17 (2) (2018) 325-345.

[15] C.R. Farrar, K. Worden, M.D. Todd, G. Park, J. Nichols, D.E. Adams, M.T. Bement, M.T. Farinholt, Nonlinear system identification for damage detection. Technical report, Los Alamos National Laboratory, 2007.

[16] K. Worden, C.R. Farrar, J. Haywood, M. Todd, A review of nonlinear dynamics applications to structural health monitoring, Struct. Control Health Monit. 15 (4) (2008) 540-567.

[17] J.M. Nichols, M.D. Todd, Nonlinear features for SHM applications, in: Encyclopedia of Structural Health Monitoring, John Wiley \& Sons, Ltd, 2009

[18] G. Kerschen, K. Worden, A.F. Vakakis, J.C. Golinval, Past, present and future of nonlinear system identification in structural dynamics, Mech. Syst. Signal Process. 20 (3) (2006) 505-592.

[19] T.J. Johnson, D.E. Adams, Transmissibility as a differential indicator of structural damage, Journal of Vibration and Acoustics-transactions of the Asme 124 (October (4)) (2002) 634-641.

[20] M. Haroon, D.E. Adams, Time and frequency domain nonlinear system characterization for mechanical fault identification, Nonlinear Dyn. 50 (November (3)) (2007) 387-408.

[21] Z.Q. Lang, Z.K. Peng, A novel approach for nonlinearity detection in vibrating systems, J. Sound Vib. 314 (July (3-5)) (2008) 603-615.

[22] Z.Q. Lang, G. Park, C.R. Farrar, M.D. Todd, Z. Mao, L. Zhao, K. Worden, Transmissibility of non-linear output frequency response functions with application in detection and location of damage in MDOF structural systems, Int. J. Non-linear Mech. 46 (6) (2011) 841-853.

[23] D.E. Adams, C.R. Farrar, Application of frequency domain ARX features for linear and nonlinear structural damage identification, Smart Nondestructive Evaluation for Health Monitoring of Structural and Biological Systems, vol. 4702 (2002) 134-147.

[24] D.E. Adams, C.R. Farrar, Classifying linear and nonlinear structural damage using frequency domain ARX models, Struct. Health Monit. - Int. J. 1 (2) (2002) 185-201.

[25] L. Bornn, C.R. Farrar, G. Park, K. Farinholt, Structural health monitoring with autoregressive support vector machines, J. Vib. Acoust. Trans. ASME 131 (April (2)) (2009) 021004

[26] L. Bornn, C.R. Farrar, G. Park, Damage detection in initially nonlinear systems, Int. J. Eng. Sci. 48 (October (10)) (2010) 909-920.

[27] M. Rébillat, R. Hajrya, N. Mechbal, Nonlinear structural damage detection based on cascade of Hammerstein models, Mech. Syst. Signal Process. 48 $(1-2)(2014) 247-259$.

[28] N. Antonin, L. Simon, P. Lotton, F. Kadlec, Modeling of nonlinear audio systems using swept-sine signals: application to audio effect, Proc. of the 12th Int. Conference on Digital Audio Effects, pages DAFx-09 (2009).

[29] M. Rébillat, R. Hennequin, É. Corteel, B.F.G. Katz, Identification of cascade of Hammerstein models for the description of nonlinearities in vibrating devices, J. Sound Vib. 330 (February (5)) (2011) 1018-1038.
[30] A. Novak, B. Maillou, P. Lotton, L. Simon, Nonparametric identification of nonlinear systems in series, IEEE Trans. Instrum. Meas. 204 (2014) 4-2051.

[31] M. Rébillat, K. Ege, M. Gallo, J. Antoni, Repeated exponential sine sweeps for the autonomous estimation of nonlinearities and bootstrap assessment of uncertainties, Proc. Inst. Mech. Eng. C: J. Mech. Eng. Sci. 230 (April (6)) (2016) 1007-1018

[32] D.A. Tibaduiza, L.E. Mujica, J. Rodellar, Damage classification in structural health monitoring using principal component analysis and self-organizing maps, Struct. Control Health Monit. 130 (2013) 3-1316.

[33] M. Rébillat, C.B. Barthes, N. Mechbal, K.M. Mosalam, Structural health monitoring of high voltage electrical swich ceramic insulators in seismic areas, in: 7th European Workshop on Structural Health Monitoring, Nantes, 2014, pp. 2183-2190.

[34] E. Balmès, SDTools, vibration software and consulting, 2016 http://www. sdtools.com/.

[35] É. Gay, L. Berthe, M. Boustié, M. Arrigoni, M. Trombini, Study of the response of CFRP composite laminates to a laser-induced shock, Compos. Part B: Eng. 64 (2014) 108-115.

[36] M. Ghrib, M. Rébillat, N. Mechbal, G. Vermot des Roches, Automatic damage quantification using signal based and nonlinear model based damage sensitive features, The 20th World Congress of the International Federation of Automatic Control (2017)

[37] M. Bakir, M. Rébillat, N. Mechbal, Damage type classification based on structures nonlinear dynamical signature, in: 9th IFAC symposium on Fault Detection, Supervision and Safety of Technical Processes, Paris, 2015, pp. 652-657.

[38] N. Cristianini, J. Shawe-Taylor, An Introduction to Support Vector Machines: And Other Kernel-based Learning Methods, Cambridge University Press, 2000.

[39] E. Bernhard, Boser, M. Isabelle, V.N. Guyon, Vapnik, A training algorithm for optimal margin classifiers, in: Proceedings of the Fifth Annual Workshop on Computational Learning Theory, ACM, New York, NY, USA, 1992, pp. 144-152.

[40] V.N. Vapnik, Statistical Learning Theory, Wiley-Interscience, 1998.

[41] S. Canu, Y. Grandvalet, V. Guigue, A. Rakotomamonjy, SVM and Kernel Methods Matlab Toolbox, 2005 http://asi.insa-rouen.fr.

[42] I.T. Jolliffe, Principal Component Analysis, Springer Verlag, 1986.

[43] M. Barker, W. Rayens, Partial least squares for discrimination, J. Chemom. Soc. 17 (3) (2003) 166-173.

[44] R. Rosipal, L.J. Trejo, B. Matthews, Kernel PLS-SVC for linear and nonlinear classification, Proceedings of the 20th International Conference on Machine Learning (ICML-03), vol. 64 (2003) 640-647.

[45] R. Rosipal, N. Krämer, Overview and recent advances in partial least squares, in: International Statistical and Optimization Perspectives Workshop "Subspace, Latent Structure and Feature Selection", Springer, 2005, pp. 34-51.

[46] J. Arenas-Garcia, K.B. Petersen, G. Camps-Valls, L.K. Hansen, Kernel multivariate analysis framework for supervised subspace learning: a tutorial on linear and kernel multivariate methods, IEEE Signal Process. Mag. 30 (4) (2013) 16-29.

[47] M. Rebillat, M. Schoukens, Comparison of least squares and exponential sine sweep methods for parallel Hammerstein models estimation, Mech. Syst. Signal Process. 104 (2018) 851-865. 